IFIT3 produced an opposite effect. Mechanistically, IFIT3 protein was found to interact both with STING and TBK1.

Conclusions We proposed that cGAS-STING signalling pathway was hyperactive in monocytes of SLE. IFIT3 is one of the important genes which contributed to the over-activation of cGAS-STING signalling pathway and over-produced IFN in SLE pathogenesis.

\section{MASS SPECTROMETRIC SEQUENCING OF PRECIPITATING ANTI-RO REVEALS UNIQUE VH/VL PEPTIDE BIOMARKERS}

${ }^{1} \mathrm{JJ}$ Wang*, ${ }^{1} \mathrm{M}$ Al kindi, ${ }^{1} \mathrm{~A}$ Colella, ${ }^{1} \mathrm{~L}$ Dykes, ${ }^{1} \mathrm{M}$ Jackson, ${ }^{2} \mathrm{~T}$ Chataway, ${ }^{3} \mathrm{~J}$ Reed, ${ }^{1} \mathrm{~T}$ Gordon. ${ }^{1}$ Flinders University, Immunology, Adelaide, Australia; ${ }^{2}$ Flinders University, Flinders Proteomic Facility, Adelaide, Australia; ${ }^{3}$ Garvan Institute of Medical Research, Immunology Division- Immunogenomics Laboratory, Sydney, Australia

\subsection{6/lupus-2017-000215.330}

Background and aims Autoantibodies directed against the 60$\mathrm{kD}$ Ro (Ro60)/SSA ribonucleoprotein particle are the major target of humoral autoimmunity in patients with systemic lupus erythematosus (SLE) and primary Sjögren's syndrome (SS). However, little is known of the anti-Ro60 immunoglobulin variable-region $(\mathrm{IgV})$ repertoire in terms of clonality and $\mathrm{IgV}$ gene usage at the level of the serum proteome.

Methods We used high-resolution mass spectrometry to sequence precipitating anti-Ro60 proteomes from sera of patients with SLE and primary SS and compare $\operatorname{IgV}$ peptide signatures in Ro/La autoantibody subsets. Anti-Ro60 were purified by elution from native Ro60-coated ELISA plates and subjected to combined de novo amino acid sequencing and database matching. Additionally, Ro60 precipitins from counterimmunoelectrophoresis gels were excised, digested and sequenced directly by mass spectrometry.

Results Anti-Ro60 Igs purified from ELISA plates and Ro60 precipitins were comprised dominant public sets of IgG1 kappa and lambda restricted heavy and light chains (with sharing of IGHV3-23, IGHV3-74 and IGHV1-18; IGKV3-20, IGKV1-5 and IGLV3-19). Significantly, mass spectrometric sequencing of purified anti-Ro60 IgGs from SLE patients showed the same convergence of autoantibody repertoires as primary SS, apart from one SLE patient who lacked IGHV374 , suggesting that humoral anti-Ro60 molecular signatures are conserved across these two systemic autoimmune diseases. Specific IgV amino acid substitutions stratified anti-Ro60 from anti-Ro60 plus anti-La responses, providing a molecular fingerprint of Ro60/La determinant spreading.

Conclusions Unique anti-Ro60 $\mathrm{IgV}$ peptide signatures provide insights in to mechanisms of autoantibody production as well as holding promise as serum-based molecular markers for clinical syndromes linked to Ro60 autoimmunity.

\section{IL-1B AND IL-6 ARE HIGHLY EXPRESSED IN RF+IGE+ SYSTEMIC LUPUS ERYTHEMATOUS SUBTYPE}

${ }^{1} Y W_{u^{*}},{ }^{1} \mathrm{~B}$ Cai, ${ }^{1} \mathrm{~J}$ Zhang, ${ }^{1} \mathrm{~B}$ Shen, ${ }^{1} \mathrm{Z}$ Huang, ${ }^{2} \mathrm{C}$ Tan, ${ }^{3} \mathrm{CC}$ Baan, ${ }^{1} \mathrm{~L}$ Wang ${ }^{*} .{ }^{1}$ West China Hospital affiliated Sichuan University, Department of Laboratory Medicine, Chengdu, China; ${ }^{2}$ West China Hospital affiliated Sichuan University, Department of Rheumatology, Chengdu, China; ${ }^{3}$ West China Hospital affiliated Sichuan University, Department of Laboratory Medicine, Rotterdam, Netherlands Antilles
Background and aims Systemic lupus erythematous (SLE) is anautoimmune disease with great heterogeneity in pathogenesis and clinical symptoms. To better categorise SLE subtypes we determined the dominant cytokines based on $\mathrm{RF}+\mathrm{IgE}+$ (both $\mathrm{RF}$ and IgE were positive ) familial SLE.

Methods RF, IgE and multiple cytokines (i.e., IL-1 $\beta$, IL-6, IL8 , IL-10, IL-17, IFN- $\gamma$, IP-10, MCP-1 andMIP-1 $\beta$ ) were measured in sera of familial SLE $(n=3)$, non-inherited SLE $(n=108)$ and healthy controls $(\mathrm{n}=80)$.

Results Three SLE patients in family and 5 out of 108 noninherited patients featured with $\mathrm{RF}+\mathrm{IgE}+$. These $\mathrm{RF}+\mathrm{IgE}$ + SLE patients expressed significantly higher levels of IL-1 $\beta$ and IL-6 than the other SLE patients $(\mathrm{p}<0.05)$. IL-6 correlated with both $\operatorname{IgE}$ and IL-1 $\beta$ levels in $\mathrm{RF}+\mathrm{IgE}+\mathrm{SLE}$ patients $\left(\mathrm{r}^{2}=0.583, \mathrm{p}=0.027 ; \mathrm{r}^{2}=0.847, \mathrm{p}=0.001\right)$.

Conclusions Both IL-1 $\beta$ and IL- 6 are highly expressed cytokines in $\mathrm{RF}+\mathrm{IgE}+\mathrm{SLE}$ subtype which may be related to the pathogenesis of this special SLE subtype.

\section{CEREBROSPINAL FLUID UBIQUITIN CARBOXYL HYDROLASE L1 (UCH-L1) AND ITS AUTOANTIBODY ARE USEFUL BIOMARKERS FOR NEUROPSYCHIATRIC SYSTEMIC LUPUS ERYTHEMATOSUS (NPSLE)}

${ }^{1} \mathrm{X} \mathrm{Li}^{*},{ }^{2} \mathrm{Y}$ Meng, ${ }^{1} \mathrm{Y}$ Gan, ${ }^{1} \mathrm{G}$ Wang, ${ }^{1} \mathrm{R} \mathrm{Mu},{ }^{1} \mathrm{X}$ Liu, ${ }^{1} \mathrm{X}$ Sun, ${ }^{1} \mathrm{Z} \mathrm{Li} .{ }^{1}$ peking university people's hospital, rheumatology and immunology, beijing, China; ${ }^{2}$ The Fifth Affiliated Hospital of Zhengzhou University, Department of Rheumatology and Immunology, Zhengzhou, China

\subsection{6/lupus-2017-000215.332}

Background and aims To identify cerebrospinal fluid (CSF) biomarkers for the diagnosis and disease severity evaluation of neuropsychiatric systemic lupus erythematosus (NPSLE).

Methods CSF samples (36 NPSLE, 19 SLE controls, 4 other connective tissue disease controls and 10 other nervous system disease controls) and serum samples (21 NPSLE and 6 SLE controls) were included in this study. The levels of UCH-L1 and its autoantibody were determined by Luminex multiplex (xMAP) assays and enzyme-linked immunosorbent assay (ELISA) respectively.

Results 1) Among 6 candidate neurological disease related proteins, including ubiquitin carboxyl hydrolase L1 (UCH-L1), total Tau protein, phospho-Tau protein, DJ-1 protein, nerve growth factor (NGF) and $\alpha$-Synuclein $(\alpha-S Y N)$, UCH-L1 was significantly elevated in the CSF of patients with NPSLE defined by 2001 Ainiala's modified criteria, while it was lower in those defined by 1999 ACR criteria but merely presented with headache or mild mood disorder, and in SLE controls whose neuropsychiatric manifestations were not due to SLE. The elevation of CSF UCH-L1 levels were associated with elevated SLEDAI and the number of NPSLE manifestations diagnosed in individual patients. 2) The CSF levels of UCH-L1 autoantibodies were significantly elevated in patients with NPSLE, and showed a sensitivity of $53 \%$ and a specificity of $91 \%$ for the diagnosis of NPSLE. CSF anti-UCH-L1 levels were associated with organ involvements, and were positively correlated with serum anti-UCH-L1 levels in the NPSLE patients.

Conclusions Anti-UCH-L1 is a promising CSF biomarker for NPSLE diagnosis with high sensitivity and specificity, and CSF levels of UCH-L1 may reflect the severity of NPSLE. 


\section{IN VIVO THERAPEUTIC SUCCESS OF MICRORNA-155 (MIR-155) ANTAGOMIR IN A MOUSE MODEL OF LUPUS ALVEOLAR HAEMORRHAGE}

Y Wang*, S Zhou, N Shen. RenJi Hospital- School of Medicine- Shanghai JiaoTong University, Department of Rheumatology, Shanghai, China

\subsection{6/lupus-2017-000215.333}

Background and aims Diffuse alveolar haemorrhage (DAH) is a rare but life-threatening complication of systemic lupus erythematosus (SLE). Pristane-treated B6 mice develop severe DAH within 2 weeks of treatment. MicroRNA-155 (miR-155) is a pleiotropic microRNA that plays a crucial role in the regulation of immune responses. The purpose of this study was to examine the role of miR-155 in the development of DAH in pristane-induced lupus using miR-155-knockout (miR-155-/-) mice and miR-155 antagomir to silence miR-155.

Methods DAH was induced by an intraperitoneal injection of $0.5 \mathrm{~mL}$ of pristane. MiR-155 antagomir was intravenously administrated to silence miR-155 expression. Lung tissues were collected for RNA extraction and were embedded in paraffin for sectioning. Gene expression profiling data were analysed using Ingenuity Pathway Analysis. Real time q-PCR was used for single validation. Luciferase reporter assay and RNAAgo2 immunoprecipitation were performed for target validation.

Results MiR-155 expression was significantly increased in the development of DAH. Disease progression was reduced in miR-155/- mice and by in vivo silencing of miR-155 using miR-155 antagomir. MiR-155 silencing dampened pristaneinduced ectopic activation of multiple inflammatory pathways, and reduced the expression of pro-inflammatory cytokines. Several negative regulators of nuclear factor (NF)- $\mathrm{\kappa B}$ signalling

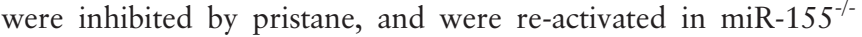
mice. In particular, the anti-inflammatory factor peroxisome proliferator-activated receptor- $\alpha$ was identified as a direct target of miR-155.

Conclusions MiR-155 promotes pristane-induced lung inflammation. MiR-155 contributes to ectopic activation of NF- $\kappa \mathrm{B}$ signalling pathways by targeting multiple negative regulators. MiR-155 antagomir may be a promising therapeutic strategy for treating acute lung inflammation in lupus.

\section{SERUM IL-18 AS BIOMARKER IN PREDICTING LONG TERM RENAL OUTCOME AMONG PEDIATRIC-ONSET SYSTEMIC LUPUS ERYTHEMATOSUS PATIENTS}

JL ${ }^{1} \mathrm{KW}$ Yeh*, 'CY Wu, ${ }^{2} \mathrm{HY}$ Yang, ${ }^{1} \mathrm{~J} \mathrm{~L} .{ }^{1}$ Chang Gung Memorial Hospital, Paediatrics, Taoyuan, Taiwan R.O.C; ${ }^{2}$ Chang Gung Memorial Hospital, Nephrology, Taoyuan, Taiwan R. O.C

\subsection{6/lupus-2017-000215.334}

Background and Aims An urge of biomarker identification is needed to better monitor lupus nephritis (LN) disease activity, guide clinical treatment, and predict patient's long-term outcome. With the proinflammatory effect and its association with inflammasomes, the significance of nterleukin-18 (IL-18) among pediatric-onset systemic lupus erythematous (pSLE) patient.

Methods In a pSLE cohort of 96 patients with an average follow-up period of $10.39 \pm 3.31$ years, clinical data and laboratory workups including serum IL-18 were collected at time of disease onset and 6 months after treatment despite their initial renal status. Through Cox regression analysis, the parameters at baseline and at 6 months posttreatment were carefully analysed.

Results Average age of all cases was 12.74 \pm 3.01 years old and 65 of them underwent renal biopsy at the time of diagnosis. Nine $(9.38 \%)$ progressed to end-stage renal disease (ESRD) and $2(2.08 \%)$ died during follow-up. Through multivariate analysis, serum IL-18 level 6 months posttreatment was found to be the most unfavourable factor associating poor clinical outcome despite patient's initial renal status. The presentation of serum IL-18 in its correlation with SLE global disease activity as well as the presence and severity of LN were all significant $(p<0.001, p=0.03$, and $p=0.02$, respectively). The histological classification of $\mathrm{LN}$ was not associated with the level of IL-18 among the pSLE patients $(p=0.64)$. Conclusions The role of serum IL-18 as biomarker representing global disease activity and status of renal flares among pSLE population was shown for the first time. Additionally, we have identified IL-18 at 6 months posttreatment a novel marker for long-term renal outcome prediction.

\section{MICRORNA-21 IS A CRITICAL REGULATOR OF AUTOIMMUNITY THROUGH PROMOTING EFFECTOR AND METABOLIC FUNCTION OF PATHOGENIC TH17 CELLS}

X Yu*, D Dai, N Shen. RenJi Hospital- School of Medicine- Shanghai JiaoTong University, Department of Rheumatology, Shanghai, China

\subsection{6/lupus-2017-000215.335}

Background and aims Systemic lupus erythematosus is a prototypical autoimmune disease that causes mortality and morbidity worldwide. Recent studies suggest proinflammatory TH17 cells are key pathogenic factors that contribute to lupus nephritis. Our group previously demonstrate that microRNA-21 was highly upregulated in $\mathrm{CD}^{+} \mathrm{T}$ cells from both lupus patients and lupus-prone mice. However, the role of microRNA-21 in pathogenic TH17 cells and they-mediated autoimmune diseases is still unclear. In this study, we systemically dissect the role of microRNA-21 in the differentiation and effector function of pathogenic TH17 cells.

Methods MicroRNA-21 knockout and conditional knockout mice were generated. EAE was induced to study the role of microRNA-21 in pathogenic TH17 cell-mediated autoimmune diseases. RNA-seq, RIP-seq and DAVID bioinformatic analysis were conducted to find key microRNA-21 regulated pathway and molecular targets in pathogenic TH17 cells. Metabolic assays were done to study the glycolytic activity of microRNA-21-deficent pathogenic TH17 cells.

Results In this study, we demonstrate that IL-6-STAT3 signalling induced microRNA-21 is essential for the late stage commitment and maintenance of pathogenic TH17 cells by targeting key regulators. MicroRNA-21-deficient TH17 cells express less pathogenic TH17 signature genes and show less glycolytic activity. Conditional deletion of microRNA-21 in $\mathrm{CD}^{+}{ }^{+} \mathrm{T}$ cells protects mice from EAE while loss of microRNA-21 expression by dendritic cells and myeloid cells do not.

Conclusions These findings suggest that microRNA-21 is a novel cell-intrinsic regulator of the commitment and metabolic function of pathogenic TH17 cells. It may be a potential therapeutic candidate with which to reprogram the immune system and help prevent and treat autoimmune diseases. 